\title{
ANO RECTAL ANOMALIES
}

\author{
By H. H. Nixon, M.A., M.B., B.Chir., F.R.C.S. \\ Consultant Paediatric Surgeon, The Hospital for Sick Children, Great Ormond Street, and Queen Mary's Hospital for \\ Children, Carshalton
}

This description is mainly based on experience gained at Great Ormond Street over the past nine years-about 300 cases having been admitted. It owes much to the teaching of Denis Browne.

The classification of Ladd and Gross (1934) into four types has been abandoned because it did not seem to give useful grouping of cases with similar origins, treatment or prognosis. A classification has been developed which is based on different conceptions of the embryology-which were first put forward by Sir Arthur Keith as long ago as 1908-and only differs in detail from the usage of Stephens (1953) and Browne (1955).

Imperforate anus is an unfortunate generic name because the great majority of cases have some opening even though it be displaced and small. Two concepts seem to explain the majority of abnormalities as clinically found. One is the idea of failure of migration so that an orifice or its remnant will remain at the site of a more primitive anus. The other is the concept of excessive fusion of the lateral genital folds ' covering' the anus and usually leaving an opening somewhere anterior to the usual anal site. (Bill, of Seattle, has recently (I958) put forward somewhat similar suggestions.)

The commoner clinical types found are:-

\section{Vulval Ectopic Anus}

Here there is an orifice below the hymen. It is usually stenotic. There may be a suggestion of dimpling at the normal anal site and there is a reasonably developed strip of muscle representing the external sphincter running back from the site of the perineal body to the region of the coccyx. More important, the puborectalis sling of the levator ani is usually normally developed around the lower end of the bowel before this takes its abnormal terminal course. Satisfactory continence can be achieved as a result of this sling.

It is believed that this condition may arise in two ways-by failure of the anus to migrate back from this primitive position or by covering of the true anus by overfusion of the lateral genital folds. This will be discussed further when treatment is considered.

\section{Perineal Ectopic Anus}

May occur in either sex, with an opening just $\vec{\omega}$ behind the scrotum in a boy or vulva in a girl. It $\bar{D}$ can be looked on as a lesser degree of the ectopia 3 . described above.

\section{Covered Anus}

In the male there is an epithelial excrescence overlying the usual anal site. It may have a thin spot in it through which 'membrane,' the meconium, shines bluish. Or the fusion may be $\vec{r}$ complete over the anal site so that a track is formed $\mathbb{D}$ running just under the skin of the midline raphe. It usually bears little epithelial pearls and opens near the root of the scrotum, but occasionally goes further along the raphe and may open as far fot ward as the coronal sulcus of the penis. The sphincter is usually fully developed in these babies. It is believed that some of the vulval 'ectopic' ani in girls also arise in this way.

\section{Microscopic Anus}

May be looked on as a variant of the above $\stackrel{2}{\overrightarrow{0}}$ covered anus. The covering may be by over- $\frac{0}{3}$ fusion of the anal tubercles in this type. Only a 'flyspeck' of meconium reveals the entrance to the bowel in an apparently imperforate perineum, but dilatation discloses a normal anus beneath.

\section{Ano-rectal Stenosis}

A fibrous ring at the upper end of the anal canal where the proctodaeum joins the rectum. Not very common, but important because it can only 음 be detected by digital examination of the rectum, $D$ and delay in diagnosis can cause such severe secondary dilatation of the rectum.

\section{Cloaca ('Imperforate Rectum ')}

A much more primitive state in which the most important practical difference from the foregoing 0 lesions is that the bowel ends above the levatoro diaphragm. It usually has an abnormal opening $\underset{\oplus}{\complement}$ which could correspond to an even more primitive $\stackrel{?}{+}$ form of anus. In the male it usually opens into the ${ }_{\vec{T}}$ prostatic urethra-the classical "atresia ani ureth- 
Fig. IA.-Vulval ectopic anus.

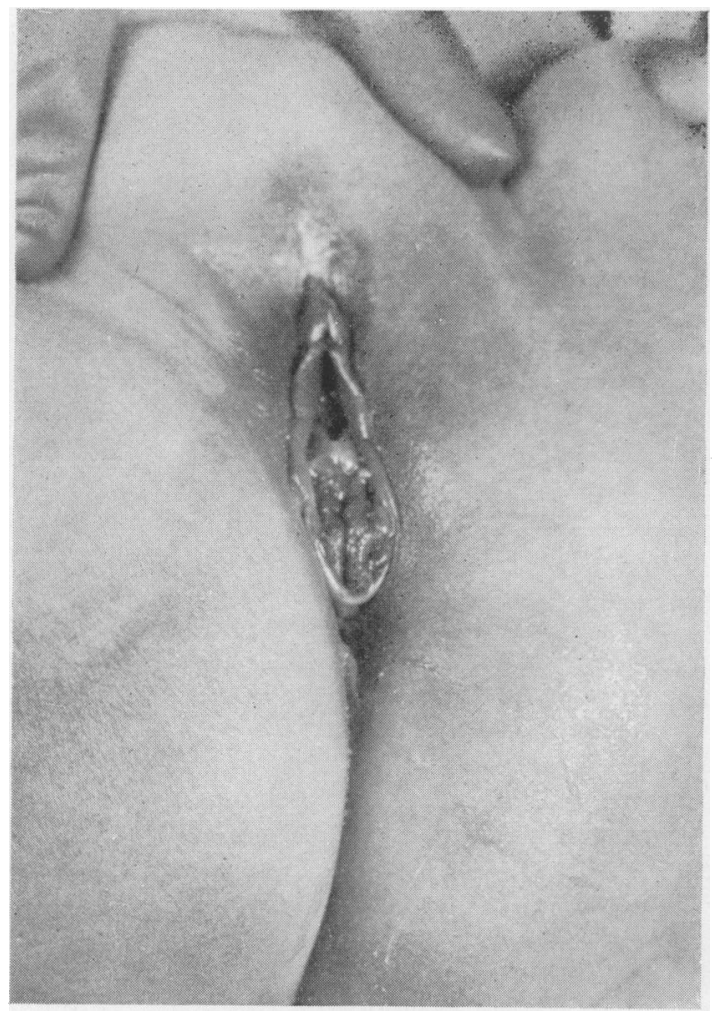

Fig. IB.-Vulval ectopic anus. The same child after 'cut back.'
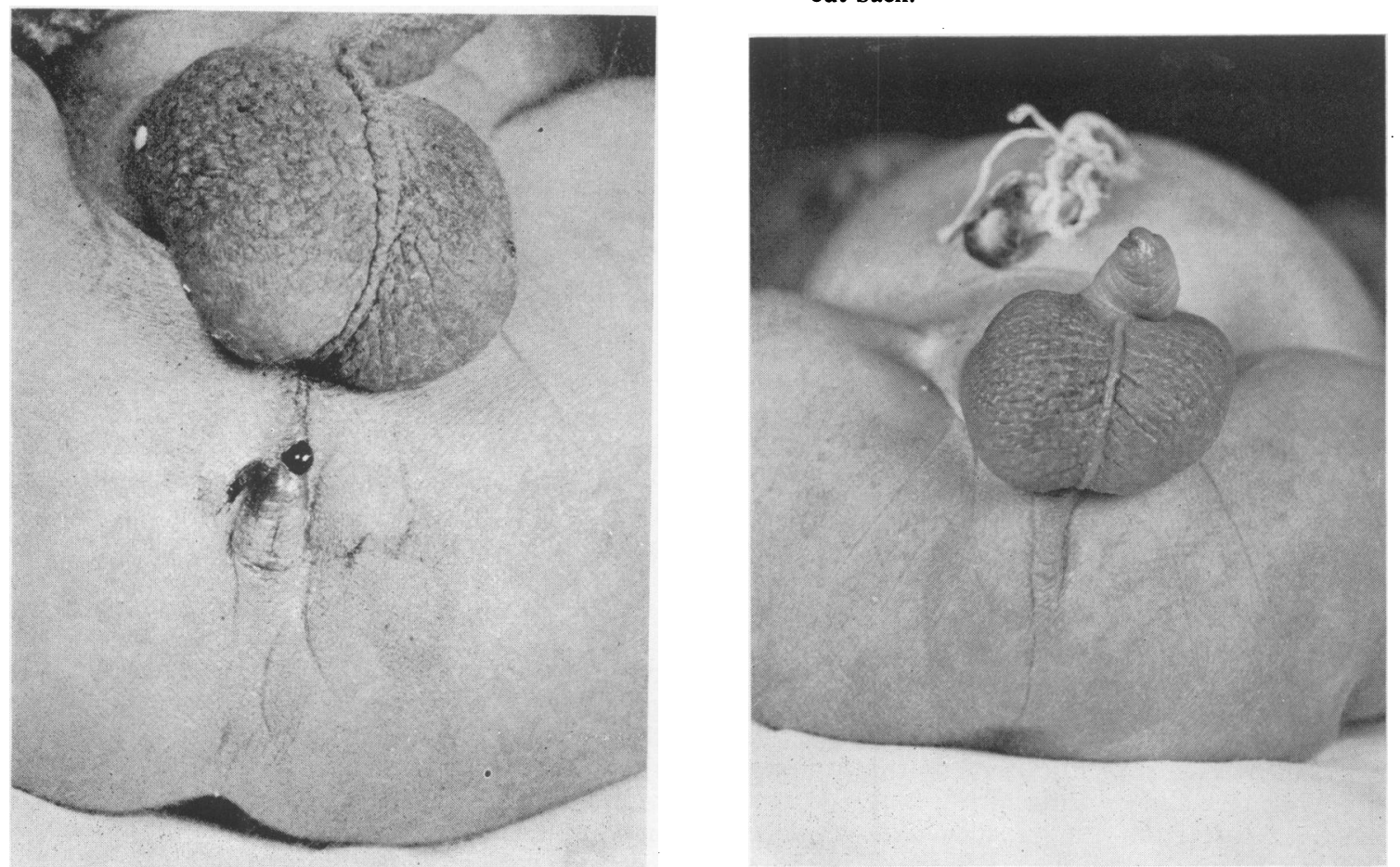

Fig. 2.- Covered anus. A bead of meconium is discharging from the orifice in front of the 'cover.'

FIG. 3.-Male 'cloaca,' i.e. ' imperforate rectum with recto-urethral fistula.' 
ralis '-though less commonly it may enter the urinary tract lower in the urethra or higher up in the bladder. (Though most of the diagnoses of ' rectovesical fistula' seem to result from failure to appreciate the height reached by the posterior urethra when the bladder is drawn out during operation in a young baby.) Thus the sign of green urine may be seen. It should be looked for early because the opening is often very small and soon blocked by debris.

In the female the abnormal opening is usually into the posterior fornix of the vagina, so that meconium may discharge from the vulva. The opening is usually small with a strong tendency to contract again after dilatation. It cannot be relied on as a ' safety valve' to make treatment any less urgent than in other types.

The essential difference between these ' high' or cloacal anomalies and the others already discussed is that the bowel stops on the pelvic floor and does not penetrate it.

The pelvic floor is often incompletely developed in these cases. The puborectalis sling was found to be absent in about a third of the cases examined by Stephens. The external sphincter may be rudimentary. The lower urinary tract may also be abnormal-in the female the bladder may open directly into the anterior vaginal wall farther inside than the normal meatus would lie, through failure to develop a urethra. In the male the attachment of the bowel may cause a potentially obstructive kink in the posterior urethra.

The female genital system may be bifid to a varying degree from a bicornuate uterus to a complete duplication with a septate vagina and a uterus opening into each side.

\section{Associated Defects}

The most important groups of associated defects are the local skeletal ones-usually sacral agenesis -and those of the urinary tract. Congenital lesions of the heart also occur in a significant proportion (Winkel Smith, I953).

Reduction of the sacrum to three segments or less has always been associated with bladder neck dysfunction in our experience (Innes Williams and Nixon, 1957; Parkkulainen, 1957).

Defects of the pelvic floor musculature have also been mentioned as common in the high anomalies and are obviously a danger to continence but may be considered as a part of the deformity rather than as an associated defect. They may also occur with the low anomalies.

The development of the urinary tract is closely bound up with that of the bowel so that it is not surprising that abnormalities are frequently associated. Apart from the bladder neck troubles already mentioned, upper tract lesions have been found in at least a third and will probably be found to be even commoner when they are looked for more consistently. Duplication of the kidney is common-also hydronephrosis, hypoplasia, aplasia and a congenital cystic condition. Fortunately unilateral abnormalities seem much more common than bilateral. Intravenous pyelography is now carried out in all cases. If the routine urine examination reveals infection then investigation is urgent. Otherwise better pictures are probably obtained with less irradiation by waiting six or twelve months. A lesion such as hydronephrosis seems capable of interfering with the baby's progress in the absence of frank urinary infections.

\section{Diagnosis}

Covered and ectopic anus can be recognized by inspection from the above criteria. The blank perineum, particularly if the natal cleft is obliterated, can be assumed to be associated with a high abnormality. Green urine in the male is evidence of the urethral fistula typical of the majority of this group. An inverted X-ray will confirm the level of obstruction. Stephens's (1953) method, relying on a lateral X-ray, is preferred to the method of Wangensteen and Rice, which can be misleading. A line is drawn from the pubes to the site of the coccyx. High anomalies stop abou this line. Low ones stop well below it. The bowef⿱ shadow is related to fixed points on the skeletot instead of to a skin marking which may be affected by the presence or absence of a median perineal fat pad. The radiograph also allows the development of the sacrum to be assessed. This gives valuable prognostic information regarding function.

\section{Treatment}

Some early operative treatment is needed in every case. Even though some lesions are treated by simple immediate measures with a view to definitive treatment later there is no place for ' tiding the baby over' till he is older. The newborn baby is as fit for any major surgery which may be indicated as he ever will be later. Delay may introduce formidable complications. The commonest are stretching of the rectum-leading to the rectal inertia syndrome with constipation and overflow incontinence-and chronic urinary infections.

Ectopic anus is treated by inserting one blade of a pair of scissors into the opening and guiding its passage backwards under the skin by external palpation. Then the scissors are closed to perform the ' cutback' or midline episiotomy. Closure of the wound edges is unnecessary. The end result of natural healing is supple new skin, whereas over-enthusiastic suture may allow pocketing of infection with increased fibrosis and inelasticity of 
the orifice. This minor operation is preferred to dilatation as being more promptly effective and eradicating the forward angulation at the outlet which otherwise tends to allow a ball-valve accumulation as faeces is pushed down behind the outlet. Dilatation may be adequate, however, for minor displacements in perineal ectopia. The anus should be made large enough to take a 12 Hegar sound in the neonatal period-or the fifth finger. Post-operative dilatations must be carried out daily for three months and then at increasing intervals over the next three months. Failure to insist on this full regime to avoid recurrent contracture will result in stretching of the rectum and eventual constipation of the rectal-inertia type with overflow incontinence. I usually teach the mother to pass the gloved fifth finger beyond the joint because of an impression that she is more likely to dilate properly and to be able to tell how the condition is progressing when she can feel the passage than when inserting a metal sound.

Covered anus is managed very similarly by opening the track which leads back from the tiny orifice which is present and working backwards until the track goes deep to reach the site of the anus. Epithelial excrescences here are cut off to avoid leaving irritating tags. Post-operative care is the same as for the ectopic anus. The anus appears virtually normal in later life and continence should be full.

Most of the ectopic ani finish with Browne's 'shot gun' perineum - with both barrels alongside-as in the naturally occurring perineal ectopic anus. Providing the enlargement of the anus has been adequate, function is normal except in the minority who have associated pelvic floor or sacral defects. No further treatment is required for this minor deviation from the normal. Normal childbirth can take place with this type of perineum. In a few, however, the anal orifice, though adequate in size, remains tilted into the posterior wall of the vagina so that it is difficult to clean, and vaginal soiling may occur with later repercussions. In such a case secondary transplantation of the anus to the normal site is performed. The best time is probably the fourth year. If it is done in the neonatal period a proportion will break down, forming a scarred stenotic channel. This is probably because the bowel wall is not tough enough to hold stitches against the strains of defaecation (so that it cannot be avoided by attention to atraumatic technique) as well as to inadequate blood supply (so that the mucosa always looks blue and congested after such an attempt). Proximal colostomy has been found also to be an inadequate safeguard against breakdown. The toddler period is avoided, because anal manipulations at this age are so upsetting and co-operation of the child in post-operative training will be unsatisfactory.

The technique of transplantation is to make an incision around the posterior border of the fourchette and, by scissor and separation dissection, to circumcize the ectopic anal orifice. Mobilization is then carried up outside the muscle coat for 5 to $7 \mathrm{~cm}$. A separate midline incision is then made, about $2.5 \mathrm{~cm}$. long at the normal anal sitewhich may be marked by a suggestion of a dimple or by a little thickening of the raphe. A tunnel is bluntly dissected forward to reach the mobilized bowel below the muscle of the pelvic floor. The bowel is drawn back to its new site and sutured in two layers. Several catgut sutures unite muscle and submucosa of bowel to pelvic muscle and fascia so as to keep strain off the outer layer of silk sutures from full thickness of bowel to skin. The vaginal defect should be loosely closed, and a suction drain in this space for two days may help to prevent fluid collecting. No rectal examination should be carried out for Io days to avoid disturbing the healing. A lubricated flatus tube may be passed as needed and the instillation of olive oil daily from the third day will prevent any faecal masses from forming. After Io days, daily digitation should begin and continue for three months to prevent contracture during healing. Aperients will probably be needed to give easy regular bowel actions. Training in regular attempts, whether the 'call' is felt or not, is essential. If any loading with faeces is detected in spite of this regime, washouts must be given promptly to prevent overstretching of the bowel before regular habits are established.

During the operation a wide-bore tube is inserted into the bowel and loosely stitched to it as suggested by Denis Browne. This makes handling much easier and also its withdrawal to the new position. It is important to avoid the temptation to make a complete midline incision from the abnormal site back to the desired one. Exposure is excellent and many books describe this incision, but it has been found that as healing proceeds the anterior part of it contracts, drawing the anus forward and upward until it finishes astonishingly near where it started.

Cloaca presents a different problem. Treatment is much more difficult in these cases where the bowel stops above the pelvic floor. The bowel has to be brought through the pelvic floor in front of the puborectalis sling before an orifice can be fashioned. An immediate abdomino anal pull through operation is preferred. The operation is not difficult if a unit is available accustomed to major surgery in the newborn. The mobility obtained by the abdominal approach allows sufficient slack in the bowel to prevent the risk of retraction 
mentioned in the discussion of perineal transplantation of the ectopic anus. Attempts at perineal operations on the high anomalies have no place. 'Classical' dissection up the hollow of the sacrum endangers the puborectalis sling on which all hope of continence may depend. The operation is not really one for the occasional neonatal surgeon. If all facilities are not available it is better to carry out a transverse colostomy as urgent treatment. This is preferable to iliac colostomy because it leaves the pelvis and sigmoid loop free for a later definitive operation. The latter should not be unduly delayed, because if the defunctioning is efficient enough the sigmoid loop may remain small and collapsed and not grow sufficiently to allow a pull through without tension. Also, urinary infections may arise in the usual male type with an opening into the urethra. Colostomy is also preferable as a primary measure if the baby is referred late in poor general condition.

Great stress is laid on the importance of making the channel for the bowel through the pelvic floor immediately behind the urethra in the male or the vagina in the female. This ensures that it will be in front of any puborectalis sling without the dangers of damage inherent in an attempt to dissect out the sling at this age. The pelvic splanchnics are clustered around the end of the bowel and should be avoided by keeping further back at this level. Damage to bladder control is the most serious complication of this operation. Further work on the anatomy of this area is in progress (J. E. Scott, unpublished observations). If the bladder (and vagina in the female) are drawn up out of the wound and back over the pubes then the 'fistula' comes up and is easier to separate than may be thought. Otherwise the operation is the standard one described by Rhoads (1948) and others.

\section{Results}

In the 'low' cases where the bowel passes through the pelvic floor before going wrong (ectopic and covered anus) the result is virtually normal continence in the great majority. The ' high' cases where the bowel stops on the pelvic floor (cloaca) are much less satisfactory. They can be trained to social cleanliness when old enough to co-operate but it cannot be called normal continence except in the rare variant with a complete proctodaeum present below the blind end of bowel. It is, however, preferred to a colostomy life. In my experience most children react badly to a colostomy, becoming much upset at puberty, if not before. To grow up with a colostomy is a very different matter to its acceptance by a mature developed individual. We feel that an attempt to avoid it is almost always justified.
The Colon

Duplication

Duplication of the bowel may occur throughout $\stackrel{\mathbb{D}}{\stackrel{2}{2}}$ its length including the colon (and rectum). Its $ᄃ$ form varies from a localized enteric cystic struc- $\overrightarrow{\vec{F}}$ ture to a long tubular duplication. This may even $\stackrel{?}{?}$ extend throughout the length of the colon and $\frac{1}{0}$ rectum. The lumen of the duplication may or $\frac{\bar{\omega}}{\bar{\omega}}$ may not communicate with the original bowel. $\frac{\vec{\sigma}}{\overrightarrow{0}}$ The mucosa may be that of a different part of the $\varrho$ bowel. The secretions of such ectopic mucosa may is cause ulceration of the adjacent colon. Thus the $\overrightarrow{0}$ condition may present in a variety of ways. $\mathrm{A}-\overrightarrow{-}$ symptomless abdominal swelling may be noticed. $\vec{\omega}$ Rectal bleeding may result from peptic ulceration $\frac{D}{0}$ (or stercoral ulceration). Ulcers may perforate, $\frac{0}{3}$ giving signs of local or spreading peritonitis. Obstruction may occur in the newborn or later.

Treatment is usually resection of the duplication $\overrightarrow{0}$ along with the parallel segment of bowel. The blood supply of the common wall is such that the

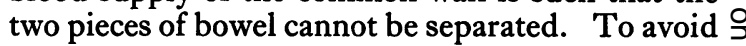
extensive resection with a very long duplication, removal of the mucosa and oversewing of the remaining wall may be considered (Waterston, personal communication). Duplication of the rectum may be treatable by making an opening loy down through the common wall so that drainage effected without removal. A difficult pelvic res section may thus be avoided.

\section{Congenital Atresia}

Congenital atresia of the colon occurs but is excessively rare. The same three types occur as in the small bowel-i.e., a septum, a cord-like segment or a complete gap. It presents clinically and radiologically as a typical large bowel obstruction in the neonatal period. The treatment consists of primary resection of the atresia and a few inches of hypertrophied bowel proximal to it, and endto-back anastomosis in the usual fashion (Nixon, 1955). If the child is grossly distended and ill when first seen a proximal colostomy is a wise primary measure.

\section{Microcolon}

Microcolon is a diagnosis to be regarded with suspicion. Any congenital abnormality which prevents the foetal colon from filling with meconium will leave it in an undeveloped looking condition-about as wide as a slate pencil. This is not $N$ an abnormal colon but an unused one-and it is capable of rapid distension and normal function when the primary lesion is treated. The latter may be a small intestinal atresia, meconium ileus or even a very long segment Hirschsprung's disease. True developmental hypoplasia or aplasia of the colon does rarely occur but the writer has 
never yet seen it without associated abnormalities making a fatal outcome inevitable.

\section{Hirschsprung's Disease}

Hirschsprung's disease almost always begins with neonatal episodes of low intestinal obstruction. They may be severe or they may be mild and transient before the classical picture of gross constipation with gaseous distension of the abdomen, an empty rectum and stunting of growth supervenes. It is the result of failure of development of the intra mural ganglia of the terminal bowel. This pathology has been present in every one of $\mathrm{I}_{22}$ consecutive cases (Wyllie, I957). Functional obstruction occurs, due to inability to produce a co-ordinated propulsive wave of contraction, and the bowel proximal to the abnormal segment dilates and hypertrophies. The affected segment consists of rectum and lower sigmoid in seven out of ten cases. The remainder may have a shorter segment involving only part of the rectum or a longer one-even extending up to the ileum. It always extends right down to the anal canal. Barium enema, using the special technique for this diagnosis, may confirm the lesion even at this early stage.

The neonatal obstruction is often relieved by routine rectal examination with the fifth finger, and a dramatic response to this procedure is very suspicious of the disease. ('Simple' meconium retention is very rare in mature babies.) If it does not deflate the baby a rectal washout may do so. If this is successful the washouts are continued daily to keep the baby fit for a Swenson operation to remore the aganglionic segment by an abdominoanal pull-through technique. If the baby remains obstructed (or if he will not thrive on washouts) then a laporotomy and colostomy should be the primary measure. It should never be done on the acutely obstructed baby until washouts have been used to try to deflate the abdomen, for this makes the procedure much less hazardous. The colostomy should be sited in the cone of transition from hypertrophied to aganglionic bowel and specimens taken for biopsy confirmation. In the rare case, where the cone appears to be proximal to the splenic flexure, ileostomy will be a wise primary measure, because the apparent cone may be lower than the true transition level. This may result from packing of meconium into the upper part of a very long segment during foetal life.

Space does not permit discussion of the further care of this condition. It has been well reviewed by Wyllie (1957).

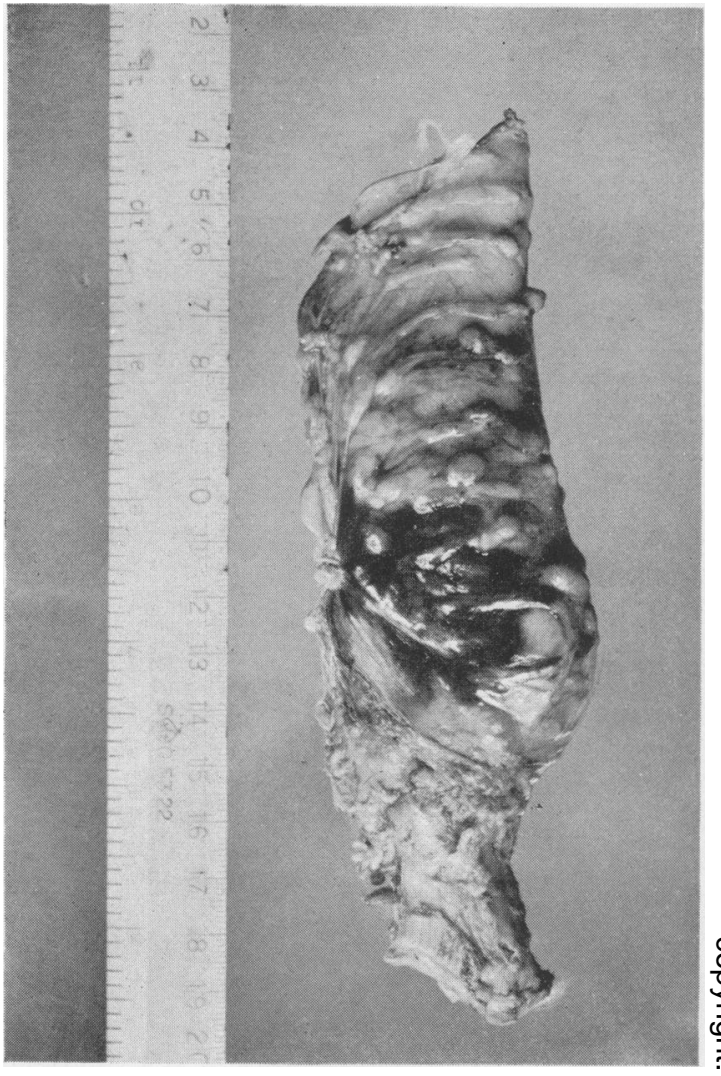

FIG. 4.-Resected specimen from a typical case of Hirschsprung's disease, aged $4 \frac{1}{2}$ months. Showing the short undilated (aganglionic) segment below the dilated and hypertrophied bowel above the pelvic floor. In this case (as in many) a resection above the pelvic floor would not have removed any of the abnormal bowel.

\section{Acknowledgement}

The photographs were supplied by Mr. Derek Martin of the Department of Medical Illustration, H.S.C., Great Ormond Street, whose help is gratefully acknowledged.

\section{BIBLIOGRAPHY}

BILL, A. H., and JOHNSON, R. J. (1958), Surg. Gynec. Obstet.,

BROW, 643. (1955),Arch. Dis. Childh. 30, 42.

WILLIAMS, D. INNES; and NIXON, H. H. (1957), Surg. Gynec. Obstet., 105, 84 .

KEITH, A. (1908), Brit. med. $\mathcal{F}$., ii, 1736.

LADD, W. E., and GROSS, R. E. (1934), Amer. F. Surg., 23, 167. NIXON, H. H. (1956), Gt. Ormond St. Ұ., I.

PARKKULAINEN, K. V. (1957), Ann. Paediat. Fenn., 3, $5 \mathrm{I}$.

RHOADS, J. E., PIPES, K. L., and RANDALL, J. PERLINGIERS (1948), Ann. Surg., 127, 552.

STEPHENS, F. D. (1953), Aust. N.Z. 尹. Surgery, 22, 16r.

STEPHENS, F. D. (1953), Ibid., 23, 9.

SMITH, C. C. WINKEL'(1953), Acta. chir. scand., 105, 305.

WYLLIE, G. G. (1957), Lancet, i, 847 .

WYLLIE, G. G. (1957), Ibid., i, 850 . 